\title{
Estudio piloto sobre la alfabetización física en niños con edades comprendidas entre 8 y 12 en Extremadura.
}

\section{Pilot study on physical literacy in children between 8 and 12 in Extremadura}

\author{
María Mendoza Muñoz', Claudia López García’, Juan Manuel Franco García’, Jose \\ Ignacio Calzada Rodríguez', Ángel Manuel Denche Zamorano', Jorge Carlos-Vivas.
}

${ }^{1}$ Facultad de Ciencias del Deporte, Universidad de Extremadura, España.

\section{Resumen}

Objetivos: Evaluar la alfabetización física mediante el Canadian Assessment of Physical Literacy (CAPL-2), en menores extremeños con edades comprendidas entre 8 y 12 años, establecer diferencias significativas entre sexos y establecer el nivel de alfabetización física de los niños/as extremeños estudiados con respecto a los valores de referencia canadienses. Material y métodos: En el estudio participaron 39 menores de entre 8 y 12 años (19 chicos y 20 chicas). La alfabetización física fue evaluada mediante el CAPL-2 con sus 4 dominios (Actividad física diaria, competencia física, motivación y confianza y conocimiento y comprensión). Resultados y discusión: Los resultados muestran que no existen diferencias significativas entre sexos para ningún dominio del CAPL-2, así como para ninguna de las pruebas incluidas en cada dominio. Además, se encontró un nivel de alfabetización física mayor en la muestra extremeña, respecto a los valores de referencia canadienses. Conclusiones: Se pudo afirmar que no existieron diferencias entre sexos para ninguno de los dominios de alfabetización física, así como una elevada alfabetización física de la muestra, respecto a los valores de referencia canadienses.
Fecha de recepción:

29-07-2020

Fecha de aceptación:

$05-11-2020$

Palabras clave
Actividad física;
alfabetización física; CAPL-
2; dominios.
Keywords
CAPL-2; domains; physical
activity; physical literacy.

\section{Abstract}

Objectives: To assess physical literacy through the Canadian Assessment of Physical Literacy (CAPL-2) in Extremadura children aged 8-12 years, to establish significant differences between sexes and the level of physical literacy of Extremadura children compared to Canadian reference values. Material and methods: The study involved 39 children between 8 and 12 years old (19 boys and 20 girls). Physical literacy was assessed through the CAPL-2 with its 4 domains (Daily physical activity, physical competence, motivation and confidence, and knowledge and understanding). Results and discussion: The results show that there are no significant differences between the sexes for any of the CAPL-2 domains, as well as for any of the tests included in each domain. In addition, a higher level of physical literacy was found in the Extremadura sample than in the Canadian reference values. Conclusions: It could be stated that there were no differences between the sexes for any of the physical literacy domains, as well as a high level of physical literacy in the sample, with respect to the Canadian reference values 


\section{Introducción}

La inactividad física es inherente a todos los países (Iraheta \& Bogantes, 2020). La transición al postmodernismo cada día aumenta el sedentarismo y limita las posibilidades de movimiento, esto es evidente en la niñez y la adolescencia, donde se han sustituidos los juegos que implicaban movimiento, por horas de pantalla, tiempo dedicado a teléfonos inteligentes, tabletas, televisiones y ordenadores, condición que lleva al aumento del sobrepeso, obesidad, problemas relacionados con la atención, ansiedad, autoestima, depresión y alteraciones del sueño (Iraheta \& Bogantes, 2020).

La inactividad física se trata de un fenómeno global que se extiende a un ritmo acelerado por todo el mundo. La actividad física es importante para los niños porque está directamente relacionada con su salud, ya que mejora la función cardiorrespiratoria, el control de la presión sanguínea y contribuye al mantenimiento del peso ideal (Bonilla, 2016). La Organización Mundial de la salud (OMS) (2010), expone que un 60\% de la población mundial no realiza la actividad física necesaria para obtener beneficios en la salud, situación por la cual es considerada el cuarto factor de riesgo de mortalidad, que causa la muerte de 32 millones de personas cada año, siendo principal causante del padecimiento de las Enfermedades No Transmisibles (ETN), las cuales evolucionan lentamente y son responsables de los altos costos de los sistemas de salud pública (García \& González-Jurado, 2017).

En diferentes informes como "The heavy burden of obesity" de La Organización para la Cooperación y el desarrollo Económico (OCDE) analizan el impacto económico, social y en salud el sobrepeso y la obesidad presentando el dato de un total de 52 países en 2016 (36 países de la OCDE, países de la UE 28 y países del G 20) (Vuik, Lerouge, Guillemette, Feigl, \& Aldea, 2019) colocando a España en la posición número 12. En esta línea, son múltiples los estudios que han contemplado el índice de sobrepeso y obesidad a lo largo de los últimos años (Gómez, Lorenzo, Ribes, \& Homs, 2019; Pérez-Farinós et al., 2013; Wijnhoven et al., 2014), siendo, en 2017, la prevalencia de sobrepeso y obesidad en España en niños de entre 2 y 17 años, de un 28,6\% según la Encuesta Nacional de Salud Española (ENSE) (Ministerio de Sanidad Servicios Sociales e lgualdad).

Además, para una salud óptima, se recomienda que los niños realicen un mínimo de 60 minutos de actividad física de intensidad moderada a vigorosa todos los días, y actividades recomendadas para el fortalecimiento de huesos al menos 3 días por semana (Mark Stephen 
Tremblay, Colley, Saunders, Healy, \& Owen, 2010). Investigaciones recientes sugieren que muy pocos niños logran este nivel recomendado de actividad física diaria (Colley et al., 2017).

Debido a los datos que arrojan estas investigaciones, surge desde la Oficina Regional para Europa de la OMS, la iniciativa Chilhood Obesity Surveillance Intiative (COSI) (Wijnhoven et al., 2014) para la vigilancia de la obesidad infantil en Europa, siendo su objetivo principal el establecer un sistema de vigilancia armonizado, con una metodología común en todos los países participantes, tratando de recabar datos antropométricos, información de factores familiares, ambientales y estilo de vida de los escolares con edades comprendidas entre 6 y 9 años. Posteriormente, existen diferentes oleadas de estudios como es el ALADINO realizado en España, ajustándose al protocolo de iniciativa COSI (Pérez-Farinós et al., 2013).

Siguiendo esta línea surge la alfabetización física, la cual podemos considerar como una evaluación integral, que permite la vigilancia de la población con respecto a los beneficios para la salud y riesgos de involucrarse en actividad física, así como el monitoreo de los resultados educativos, es decir, mejorando la comprensión de la participación de los niños en la actividad física y motivación y confianza agregadas, conocimiento y comprensión y competencia física para la actividad física que nos permitirá apoyar mejor el desarrollo de los niveles más altos de alfabetización física infantil. Su logro permitirá la participación en una actividad física estructurada, adoptando un estilo de vida activo a edades tempranas el cual puede continuar hasta la adultez (Bonilla, 2016), y con ello conseguir números beneficios para la salud. Hay pruebas de que los patrones de conducta en cuanto a la actividad física en la infancia permanecen en la vida adulta (Twisk, Kemper, \& Van Mechelen, 2000) si además de todo ello, tenemos en cuenta la dificultad de modificar los hábitos en la vida adulta, podemos decir sin temor a equivocarnos que la infancia y la adolescencia son etapas clave en la prevención primaria de las enfermedades cardiovasculares y otras enfermedades asociadas al sedentarismo (Martínez-Vizcaíno \& Sánchez-López, 2008).

La alfabetización física, se definió en el Boletín del Consejo Internacional de Ciencias del Deporte y Educación Física de la UNESCO (United Nations Educational, Scientific and Organization) como motivación, confianza, competencia física, conocimiento y comprensión para valorar y participar en un estilo de vida físicamente activo (de Balazs, de D'Amico, \& Cedeño, 2017).

De la misma forma que la lectura, la escritura, la escucha y el habla se combinan para formular la alfabetización lingüística que permite una vida con lectura y comunicación, la alfabetización 
física es un viaje progresivo en el que los diferentes componentes (es decir, la competencia física, el comportamiento diario, el conocimiento y la comprensión, la motivación y la confianza) interactúan de manera integral para facilitar una vida de participación y disfrute de la actividad física (Margaret Whitehead, 2010).

Margaret Whitehead (2010), resalta que este planteamiento incorpora que en la experiencia de la vida humana es esencial estar físicamente educados a través de diferentes actividades físicas, por lo que el profesional del área tiene la oportunidad de relacionar sus conocimientos con las vivencias del estudiante de manera que fomente su desarrollo armónico e integral como instrumento para obtener salud, y, de esta manera, se inclinen hacia las actividades que sean de su agrado, lo cual beneficiará sus potencialidades y calidad de vida. Según esta autora la alfabetización física (Margaret Whitehead, 2010) se describe como la disposición, motivación, confianza, competencia física, conocimiento y comprensión para valorar y asumir la responsabilidad de realizar actividad física a nivel individual a lo largo de la vida. También se ha descrito como el físico y atributos psicológicos que son fundamentales para la participación en actividad física, y por lo tanto, la capacidad de un estilo de vida activo (Cairney \& Veldhuizen, 2017; Longmuir \& Tremblay, 2016). Para la realización de actividad física, la alfabetización física puede suponer la base para mantener la salud de las personas (Cairney, Dudley, Kwan, Bulten, \& Kriellaars, 2019). En Canadá y Estados Unidos, el reconocimiento de la importancia de alfabetización física y las limitaciones de los deportes tradicionales han contribuido a un cambio hacia la alfabetización física con currículos enriquecidos, que nutren habilidad, confianza, motivación y participación (Roetert, Kriellaars, Ellenbecker, \& Richardson, 2017).

Un niño físicamente alfabetizado puede moverse con habilidad y confianza en una gran variedad de situaciones físicamente desafiantes, puede leer el entorno físico, anticipar posibles necesidades de movimiento y puede responder de manera inteligente e imaginativa (de Balazs et al., 2017). Por lo contrario, un niño que aún no ha desarrollado un alto nivel de alfabetización física tratará de evitar la actividad física siempre que sea posible, tendrá una confianza mínima en su capacidad física y no estará motivado para participar en una actividad física estructurada (Mark S Tremblay et al., 2018). Además, es probable que el realizar menor actividad física correspondiente a la edad, aumente el riesgo de padecer problemas de salud tempranos o acelerados (Mark Stephen Tremblay et al., 2010). 
En respuesta a la necesidad de datos objetivos de alfabetización física, surge la evaluación canadiense de alfabetización física, CAPL-2 (Canadiann Assessment of Physical Literacy), protocolo completo que permite evaluar de manera precisa y fiable un amplio espectro de habilidades y capacidades que contribuyen y caracterizan el nivel de alfabetización física de un niño (Mark S Tremblay et al., 2018). De acuerdo con la definición internacionalmente aceptada de alfabetización física (de Balazs et al., 2017), el CAPL-2 fue diseñado para combinar por el grupo de investigación Healthy Active Living and Obesity Research Group (HALO) conteniendo evaluaciones de motivación y confianza, competencia física (relacionada con la salud, aptitud física y motora), conocimiento y comprensión, y participación habitual en la actividad física (actividad física y comportamientos sedentarios) (Healthy Active Living and Obesity Research Group, 2014).

De acuerdo con Whitehead (Margaret Whitehead, 2010), la motivación en la actividad física refleja "voluntad y entusiasmo" para tomar medidas que sean expresadas como una alegría de movimiento, confianza en las propias habilidades físicas, una actitud positiva hacia la participación y una expectativa de participación exitosa. La competencia física no sólo refleja competencia en movimiento sino también en capacidad (fuerza, resistencia, etc.) para el movimiento. El conocimiento y la comprensión abarcan movimiento (cómo moverse), rendimiento (evaluación-acción del movimiento), así como salud y estado físico (valor del ejercicio, necesidad de relajación, sueño, etc.).

La práctica de actividad física es determinante para la motivación del individuo hacia la realización de una determinada tarea o actividad. Los sujetos activos muestran una motivación intrínseca mayor que los sedentarios según Capdevila, Niñerola y Pintanel (2004). Por lo que, la oportunidad de fomentar la alfabetización física en la escuela como alternativa para promover una educación adecuada y disminuir la brecha existente en la actualidad con relación a la práctica habitual de actividades físicas, tiene como objetivo enseñar y evaluar conceptos y habilidades básicas para que las personas puedan utilizarlas en la vida cotidiana y desarrollas nuevas oportunidades sociales, no existiendo conflicto entre alfabetización y educación física ya que esta última es una materia escolar y la primera es un objetivo que todos podemos lograr.

La evaluación y concepto de alfabetización física hace que se proporcione un término que ayudará a promover la actividad física y combatir las consecuencias de los estilos de vida sedentarios (Spengler \& Cohen, 2015), además proporciona una terminología similar a otras 
materias escolares, un propósito común y un fundamento sólido para la educación física (Roetert \& MacDonald, 2015), y más específicamente, va a permitir consolidar un término unificador para describir el resultado general de programas de educación física, actividad física, deporte y recreación de calidad (Roetert \& Jefferies, 2014), sirviendo así como un resultado del área de educación física. Por tanto, la alfabetización física podría proporcionar una base para el deporte de élite, la salud pública y la educación física, tratando de promover esta desde todos los ámbitos y abordándola desde la etapa infantil, para que ya desde niños, puedan disfrutar de una actividad física plena, y con ello crear adherencia a las misma.

Según nuestro mejor conocimiento, en Extremadura no se ha publicado ninguna investigación sobre el nivel de alfabetización física de la población infantil, por lo que el principal objetivo del presente trabajo es evaluar la alfabetización física mediante el CAPL-2, teniendo en cuenta los diferentes dominios y dentro de cada uno de ellos, las diferentes pruebas que rigen esta evaluación en una muestra de conveniencia en menores extremeños entre 8 y 12 años. Así mismo como objetivos secundarios se plantea analizar si existen diferencias significativas entre sexos para los diferentes dominios, y diferentes pruebas dentro de cada uno de ellos y finalmente establecer el nivel de alfabetización física de los niños/as extremeños con respecto a los valores de referencia canadienses.

\section{Método}

\section{Participantes}

La muestra del presente estudio, fue una muestra por conveniencia compuesta por 39 participantes de entre 8 y 12 años. Para su reclutamiento se concertó una reunión con el personal directivo del centro C.E.I.P "Miguel Garrayo" (Fuente del Maestre, Badajoz) y el profesor responsable del área de educación física, en la cual se explicó el desarrollo de la evaluación y estos aceptaron tomar parte de la misma. Una vez obtenida la aceptación por parte del centro, el profesor responsable distribuyo un consentimiento informado a los participantes, los cuales fueron firmados por los padres de los mismos, autorizando así a sus hijos a la participación en el estudio y asintiendo de igual manera los propios participantes.

La muestra estuvo formada por 39 participantes 19 chicos (48,72\%) y 20 chicas (51,28\%), con edades comprendidas entre los 8 y 12 años, todos ellos pertenecientes al centro educativo C.E.I.P "Miguel Garrayo" (Fuente del Maestre, Badajoz). Para la selección de la muestra se comprobó si se cumplía con diferentes criterios de inclusión (Tener una edad comprendida entre 8-12 años; cumplimentación del consentimiento informado; no poseer contraindicaciones médicas o físicas para realizar actividad física; no tener limitaciones 
cognitivas que le impidieran seguir las instrucciones y participar de forma segura en el estudio; y cumplimentar los cuestionarios adecuadamente). Todos los protocolos siguieron la Declaración Helsinki, y el estudio fue aprobado por el Comité de Ética Biomédica 138/2019 de la Universidad de Extremadura.

\section{Procedimiento e instrumentos de evaluación}

Las evaluaciones fueron llevadas a cabo por dos graduados en magisterio en educación física y con formación específica en la evaluación que se desarrolla. Además, durante todo el proceso, se llevó a cabo la supervisión y el apoyo del responsable del área de educación física del centro.

Peso y altura: Se realizaron en condiciones estandarizadas, siguiendo en todo momento el protocolo establecido en el Manual de Procedimiento de Recopilación de Datos, elaborado específicamente para la iniciativa Childhood Obesity Surveillance Initiative (COSI) (Wijnhoven et al., 2014). Antes de tomar cualquiera de las medidas, se les pidió quitarse zapatos y calcetines, así como cualquier ropa pesada (abrigos, suéteres, chaquetas, etc.). También vaciaron sus bolsillos, se quitaron los cinturones y demás accesorios. La altura se midió con un tallímetro (Tanita Tantois, Tanita Corporation, Tokyo, Japon). El tallímetro se colocó previamente en una superficie vertical de manera que la escala de medida estaba perpendicular al suelo. La talla se medió en bipedestación, con los hombros equilibrados y los brazos relajados a lo largo del cuerpo. La medida se tomó en cm, hasta el mm más cercano. Por otro lado, el peso se midió con un bioimpedanciómetro (Tanita MC-780 MA, Tanita Corporation, Tokyo, Japon), se registró en kilogramos, hasta los 100 g más próximos.

Alfabetización física: Los participantes completaron las pruebas de aptitud física siguiendo la evaluación canadiense de alfabetización física (CAPL-2)(Longmuir, Gunnell, et al., 2018; Mark $S$ Tremblay et al., 2018). Dicho protocolo permitió evaluar de manera precisa y fiable un amplio espectro de habilidades y capacidades que contribuyen y caracterizan el nivel de alfabetización física de un niño, llegando más allá de la aptitud o habilidad motora de manera aislada, es decir, permite evaluar múltiples aspectos de alfabetización física.

El instrumento CAPL-2 está formado por 4 dominios (Tabla 1) (Healthy Active Living and Obesity Research Group, 2014). Estos dominios evalúan la alfabetización física general, la cual viene determinada por diferentes dominios: motivación y confianza (30 puntos), conocimiento y comprensión (10 puntos), competencia física (30 puntos) y comportamiento diario respecto a la actividad física (30 puntos). Las puntaciones que se otorgan a cada dominio mencionado se desarrollaron a través de una consulta en Delphi con expertos internacionales en alfabetización física infantil y actividad física (Francis et al., 2016). 
La puntuación numérica total y en cada dominio, informa sobre el nivel del participante (insuficiente, en progreso, suficiente y excelente) en función de la edad y sexo.

Tabla 1: Dominios y puntuación CAPL-2

A B

C D

\begin{tabular}{ccccc}
$\begin{array}{c}\text { Actividad } \\
\text { física diaria }\end{array}$ & $\begin{array}{c}\text { Competencia } \\
\text { física }\end{array}$ & $\begin{array}{c}\text { Motivación y } \\
\text { confianza }\end{array}$ & $\begin{array}{c}\text { Conocimiento y } \\
\text { comprensión }\end{array}$ & $\begin{array}{c}\text { Puntuación total } \\
\text { CAPL-2 }\end{array}$ \\
\hline 30 puntos & 30 puntos & 30 puntos & 10 puntos & 100 puntos \\
\hline
\end{tabular}

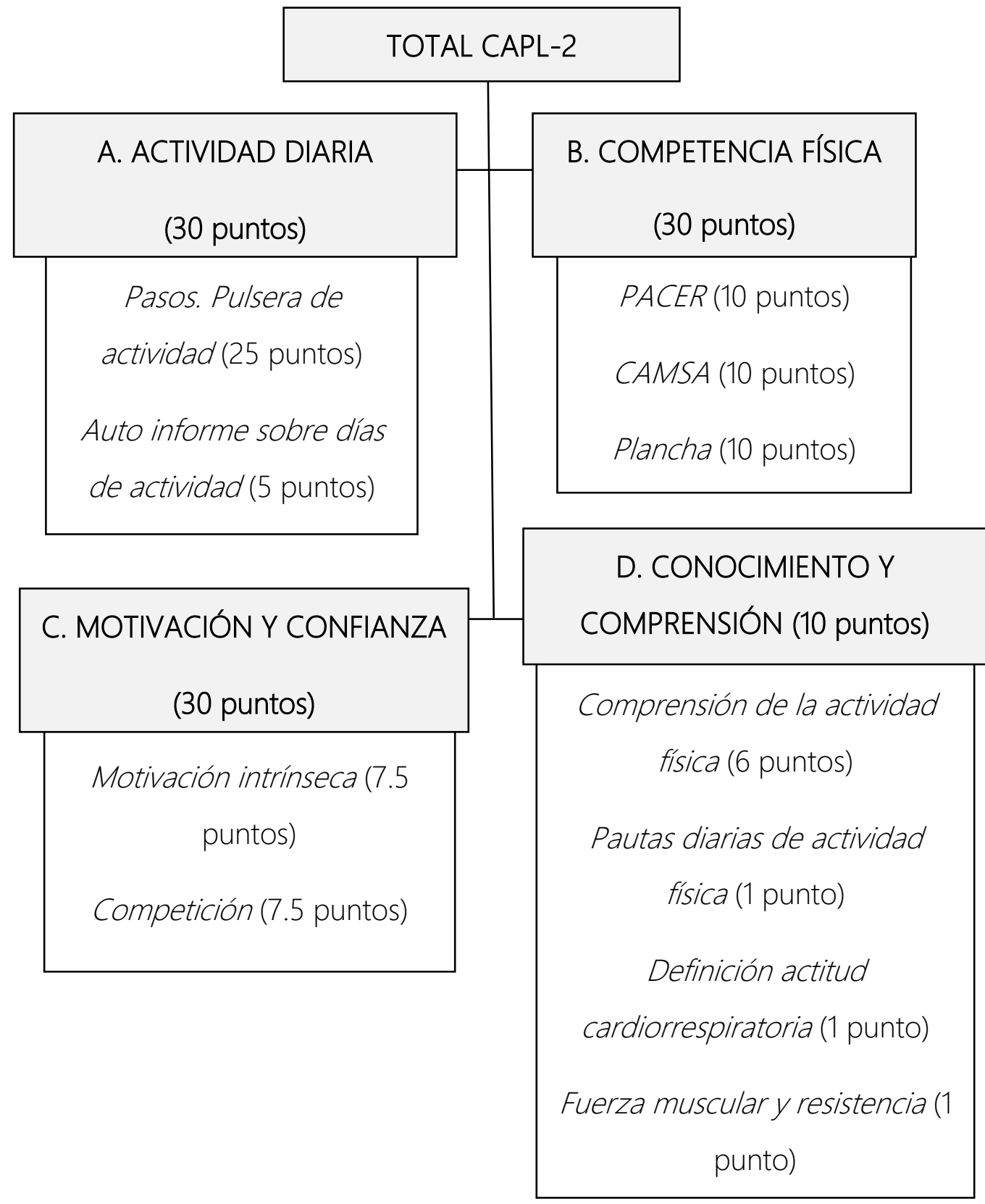

Figura 1: Puntuación total de cada dominio y de las pruebas que los componen 
Como se ha mencionado, la puntuación total, estaba dividida en cuatro dominios bien diferenciados (Actividad diaria o Dominio A, Competencia física o dominio B, Motivación y confianza o dominio C y Conocimiento y comprensión o dominio D) estando formados, cada dominio a su vez, por diferentes pruebas (Figura 1).

El dominio A o dominio de actividad física diaria, alcanzó su puntuación total con las puntuaciones de dos componentes: recuentos de pasos mediante una pulsera de actividad (Xiaomi mi Band 3) y una pregunta autoreportada sobre los minutos de actividad física semanal realizados. El valor de los pasos (25 puntos) supone un mayor peso que la pregunta sobre actividad física (5 puntos). La puntuación del componente de recuento de pasos se calcula a partir de la cantidad de pasos registrados por la pulsera de actividad y el componente de actividad física autoreportada se basó en responder a una pregunta sobre el número de días que se estuvo activo durante al menos 60 minutos.

Dominio B o dominio de competencia física, alcanza su puntuación final con la suma de las puntuaciones de tres componentes: Plancha (Boyer et al., 2013), PACER (Progresive Aerobic Cardiovascular Endurance Run), permitiendo conocer la competencia cardiorrespiratoria del niño/a a través de la prueba de Course Navette y CAMSA (Canadian Agility and Movement Sill Assessmen)(Longmuir et al., 2017), la cual permite comprobar la capacidad motora del alumnado mediante un circuito de agilidad. Todas ellas poseen una puntuación posible de 1 a 10 puntos, sumando un total de entre 1 a 30 puntos.

Ahora bien, el dominio C o dominio de motivación y confianza evalúa la confianza de un niño en su capacidad para ser físicamente activo, y su motivación para participar en la actividad física. La puntuación de este dominio es adquirida mediante la suma de diferentes puntuaciones a lo largo de 4 preguntas que corresponden con los siguientes ámbitos: parte 1: motivación intrínseca, parte 2: competición, parte 3: predilección y parte 4 adecuación; dando lugar a la puntuación total de motivación y confianza a través de una suma numérica de puntuaciones entre sus preguntas las cuales puntúan de 1 a 7.5 puntos, siendo el total del dominio de 1 a 20 puntos.

El dominio D o dominio de conocimiento y comprensión evalúa el conocimiento de la alfabetización física de un niño (Longmuir, Woodruff, Boyer, Lloyd, \& Tremblay, 2018). Similar al anterior alcanza su puntuación total a través de cinco preguntas, cuatro preguntas que responden a un apartado tipo test con un valor de 0 a 1 punto cada pregunta y por último una elección de palabras de un recuadro para rellenar huecos que faltan para completar una historia, medida esta pregunta de 1 a 6 puntos, siendo el total del dominio de 1 a 10 puntos. 
Datos perdidos

El manual CAPL-2 cuenta con información para realizar el cálculo de las puntuaciones en caso de que falte algún elemento de evaluación o la puntuación de un dominio completo. Esto permite que los resultados de la evaluación CAPL-2 se calculen o interpreten incluso si un niño no puede participar en algún protocolo de evaluación específico o si no desea completar alguna evaluación. En el estudio, fue imposible completar la prueba CAMSA o circuito de agilidad. Para sustituir los datos perdidos, se utilizaron los siguientes procedimientos como marcaba el manual:

Se realizó una división entre el total de puntos que se obtuvieron y el total posible, es decir, se eliminó la parte que no se completó. En este caso, el niño no pudo completar la prueba "CAMSA" (Valorada en 10 puntos), entonces la puntuación del dominio de competencia física sería de un total de 20 en lugar de los 30 iniciales. Si la suma de las puntuaciones del niño para los componentes del dominio de competencia física es de 16, la fracción se calculó como: $16 / 20=0,80$.

Posteriormente, la fracción se multiplicó por los máximos puntos posibles de dicho dominio, por ejemplo: los máximos puntos posibles para el dominio de competencia física eran 30 puntos. Siguiendo el caso anterior, calculamos de la siguiente manera: 0,80* $30=24$.

Con lo cual el desempeño del niño en el dominio de la competencia física se interpretó basándose en una puntuación de 24 puntos sobre 30.

Niveles de puntuaciones numéricas

Las puntuaciones numéricas de CAPL-2 completan un valor sobre 100 puntos, a los que se le asignan 4 niveles a la hora de las marcas al completar las pruebas: insuficiente, en progreso, deficiente y sobresaliente tal como indica el manual de administración del CAPL-2 o evaluación canadiense de alfabetización científica. (Tabla 2)

El nivel insuficiente y en progreso corresponde a niños que aún no han alcanzado el nivel óptimo de alfabetización física. El nivel suficiente identifica a los niños que han alcanzado un puntaje asociado con una suficiente alfabetización física. El nivel sobresaliente demuestra un alto nivel de alfabetización física. 
Tabla 2: Niveles de puntuación por categoría de interpretación

\begin{tabular}{c} 
SOBRESALIENTE \\
Supera el nivel mínimo recomendado \\
SUFICIENTE \\
Cumple el nivel mínimo recomendado \\
\hline EN PROGRESO \\
Alcanzando el nivel similar a los niños de su misma edad \\
\hline
\end{tabular}

\section{INSUFICIENTE}

Comenzando la alfabetización física, limitada comparada con los niños de su edad

La administración del CAPL-2 se llevó a cabo durante 9 días como se observa en la tabla 3.

El primer día, los niños completaron los cuestionarios de motivación, confianza, conocimiento y comprensión. Además, se les entregó y explicó el uso de la pulsera de actividad, así como su hoja de registro de pasos por día y su manual de instrucciones para su uso.

Tabla 3: Organización de tareas concurridas por orden cronológico

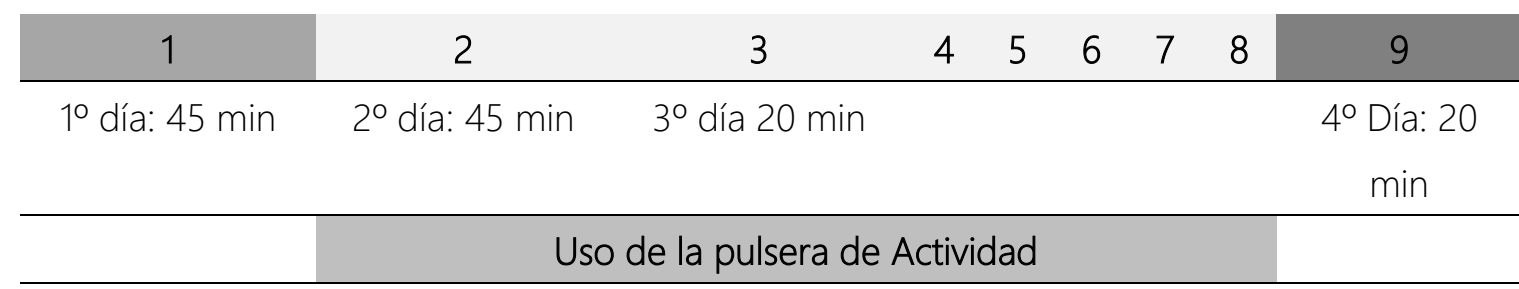

\begin{tabular}{|c|c|c|c|}
\hline $\begin{array}{l}\text { 1. Evaluación de } \\
\text { cuestionarios }\end{array}$ & $\begin{array}{l}\text { 1. Medición de } \\
\text { talla }\end{array}$ & $\begin{array}{c}\text { 1. Evaluación de } \\
\text { la prueba de }\end{array}$ & $\begin{array}{l}\text { 1. Recogida } \\
\text { de los datos }\end{array}$ \\
\hline 2. Entrega y & 2. Evaluación & resistencia & del \\
\hline explicación del & composición & aeróbica de 15 & podómetro \\
\hline uso de la & corporal & $\mathrm{m} / 20 \mathrm{~m}$ & y la hoja de \\
\hline pulsera de & & (PACER) & registro. \\
\hline actividad & & & 2. Evaluación \\
\hline Clase & & & le la \\
\hline
\end{tabular}

Plancha 
El segundo día de evaluación se evaluó la composición corporal a través del bioimpedanciometro y la medición de talla a través del tallímetro.

El tercer día, tuvo lugar la prueba de resistencia aeróbica de 25 m/20m (PACER).

Por último, tras ocho días con la pulsera de actividad, se recogieron las hojas de registro de cada alumno y el material proporcionado. Después de esto, se realizó la evaluación de la plancha en grupos reducidos.

\section{Análisis estadístico}

El análisis estadístico fue realizado mediante el software IBM SPSS Stadistics 20. En primer lugar, se realizó un análisis estadístico descriptivo para la caracterización de la muestra, en el que se reportaron los datos como media (desviación típica) y mediana (rango intercuartílico) para las variables: edad, altura, peso, dominio actividad física, dominio competencia física sin agilidad, dominio motivación, dominio conocimiento y total CAPL-2 (Canadian Assessment of Physical Literacy, segmentados por sexo (chicas y chicos).

Se realizaron también las correspondientes pruebas de normalidad mediante la aplicación del test de Shapiro-Wilk (puesto que $n<50$ para todas las muestras) para comprobar la distribución de los datos en las diferentes variables.

Para evaluar las diferencias entre grupos de sexo, se aplicó la prueba no paramétrica U de Man Whitney para las variables que no siguen una distribución normal (Total dominio actividad física diaria, puntuación de la plancha, puntuación de la prueba 15/20 m, total del dominio de competencia física sin agilidad, motivación intrínseca, competición, predilección y adecuación, así como el total del dominio motivación y confianza, las puntuaciones de las preguntas correspondientes al dominio conocimiento y comprensión y el dominio completo). La prueba paramétrica T-Student fue utilizada para aquellas variables que sí seguían una distribución normal como es el total del dominio de actividad física diaria y la altura. Las diferencias se consideraron significativas para $p$ con un valor $\leq 0,05$.

\section{Resultados}

En la tabla 4 se puede observar que entre ambos sexos no existen diferencias significativas para ninguna de las variables. Aunque en todas las variables los puntajes son similares, se puede destacar la media del dominio B o dominio de competencia física siendo mayor en los chicos que en las chicas aunque al calcular el total de todos los dominios, las chicas destacan con respecto a los chicos. 
Tabla 4. Caracterización y diferencias entre sexos.

\begin{tabular}{|c|c|c|c|c|}
\hline & & & & \\
\hline & & Chicos & Chicas & $p$ \\
\hline \multirow{3}{*}{ Edad (años) } & N (\%) & $19(48,72)$ & $20(51,28)$ & \multirow{3}{*}{$0,496^{*}$} \\
\hline & Media (DT) & $10,89(1,59)$ & $10,55(1,57)$ & \\
\hline & Mediana (RI) & $12,00(1,00)$ & $11,00(3,75)$ & \\
\hline \multirow{3}{*}{ Altura (cm) } & N (\%) & $19(48,72)$ & $20(51,28)$ & \multirow{3}{*}{, $065^{+}$} \\
\hline & Media (DT) & $145,37(11,24)$ & $141,85(9,82)$ & \\
\hline & Mediana (RI) & $145,0(10,00)$ & $141,00(10,00)$ & \\
\hline \multirow{3}{*}{ Peso (kg) } & $N(\%)$ & $19(48,72)$ & $20(51,28)$ & \multirow{3}{*}{$0,813^{*}$} \\
\hline & Media (DT) & $38,45(4,95)$ & $38,92(9,75)$ & \\
\hline & Mediana (RI) & $34,40(18,3)$ & $35,65(8,7)$ & \\
\hline \multirow{3}{*}{$\begin{array}{l}\text { Dominio A. Actividad diaria } \\
\text { (puntos) }\end{array}$} & $\mathrm{N}(\%)$ & $19(48,72)$ & $20(51,28)$ & \multirow{3}{*}{$0,369+$} \\
\hline & Media (DT) & $21,68(4,95)$ & $18,20(6,36)$ & \\
\hline & Mediana (RI) & $22,00(9,00)$ & $17,50(9,75)$ & \\
\hline \multirow{3}{*}{$\begin{array}{l}\text { Dominio B. Competencia } \\
\text { física (puntos) }\end{array}$} & N (\%) & $19(48,72)$ & $20(51,28)$ & \multirow{3}{*}{$0,285^{*}$} \\
\hline & Media (DT) & $24,80(5,00)$ & $26,20(5,78)$ & \\
\hline & Mediana (RI) & $25,50(7,50)$ & $30,00(6,00)$ & \\
\hline \multirow{3}{*}{$\begin{array}{l}\text { Dominio C. Motivación y } \\
\text { confianza (puntos) }\end{array}$} & N (\%) & 15 (50\%) & 15 (50\%) & \multirow{3}{*}{$0,813^{*}$} \\
\hline & Media (DT) & $27,28(2,95)$ & $27,25(2,33)$ & \\
\hline & Mediana (RI) & $28,50(4,7)$ & $27,30(4,65)$ & \\
\hline \multirow{3}{*}{$\begin{array}{l}\text { Dominio D. Conocimiento y } \\
\text { comprensión (puntos) }\end{array}$} & N (\%) & $19(48,72)$ & $20(51,28)$ & \multirow{3}{*}{$0,365^{*}$} \\
\hline & Media (DT) & $6,53(2,09)$ & $7,15(1,31)$ & \\
\hline & Mediana (RI) & $7,00(3,00)$ & $7,00(2,00)$ & \\
\hline \multirow{3}{*}{ Total CAPL-2 (puntos) } & $19(48,72)$ & $20(51,28)$ & $19(48,72)$ & \multirow{3}{*}{$0,902^{*}$} \\
\hline & $80,94(11,48)$ & $81,32(9,15)$ & $80,94(11,48)$ & \\
\hline & $82,10(22,7)$ & $83,00(8,8)$ & $82,10(22,7)$ & \\
\hline
\end{tabular}

CAPL-2 (Canadian Assessment of Physicial Literacy) (Los datos se muestran como media (Desviación Típica) y Mediana (Rango Intercuartílico); Las diferencias de sexo fueron analizadas según el test de U Mann-Whitney U para las variables no paramétricas $\left(p^{\star}\right)$ y el test de $T$ de Student para las variables paramétricas $\left(p^{+}\right)$.

La tabla 5, muestra las puntuaciones para el dominio de actividad diaria, así como las variables de este dominio; pasos y pregunta autorreportada sobre la actividad física diaria. Se puede observar como en las puntuaciones de los pasos destacan mayormente los chicos con respecto a las chicas mientras que en la pregunta autorreportada obtienen puntajes similares o cercanos. A la hora de visualizar la puntuación total del dominio, se ve como los chicos 
presentan mayor puntaje en el dominio de actividad física que las chicas, aunque no se aprecian diferencias significativas.

Tabla 5. Dominio A. Actividad diaria

\begin{tabular}{lcccc}
\hline & & Chicos & Chicas & p \\
\hline N (\%) & & $19(48,72)$ & $20(51,28)$ & \\
\hline \multirow{2}{*}{ Pasos (puntos) } & Media (DT) & $17,31(4,55)$ & $14,30(6,07)$ & \multirow{2}{*}{$0,120^{*}$} \\
\cline { 2 - 4 } & Mediana (RI) & $17,00(8,00)$ & $13,00(9,75)$ & \\
\hline Pregunta autorreportada & Media (DT) & $4,36(0,76)$ & $3,90(1,20)$ & \multirow{2}{*}{$0,1^{*}$} \\
\cline { 2 - 5 } (puntos) & Mediana (RI) & $5,00(1,00)$ & $4,00(1,75)$ & \\
\hline \multirow{2}{*}{ Total (puntos) } & Media (DT) & $21,68(4,95)$ & $18,20(6,36)$ & \multirow{2}{*}{$0,065^{+}$} \\
\cline { 2 - 5 } & Mediana (RI) & $22,00(9,00)$ & $17,50(6,36)$ & \\
\hline
\end{tabular}

Pasos (de 1 a 25 puntos); Pregunta autorreportada (de 1 a 5 puntos); Total (de 1 a 30 puntos); Los datos se muestran como media (Desviación Típica) y Mediana (Rango Intercuartílico); Las diferencias de sexo fueron analizadas según el test de $U$ Mann-Whitney U para las variables no paramétricas $\left(p^{\star}\right)$ y el test de T de Student para las variables paramétricas $\left(p^{\dagger}\right)$.

La tabla 6, muestra las puntuaciones medias de la Plancha y la prueba PACER que completan el dominio de competencia física. Podemos observar que dentro de la categoría de la plancha destacan más las chicas que los chicos, mientras que en la categoría PACER, destacan más los chicos que las chicas. Por lo que, si visualizamos el total del dominio, la media de las chicas está por encima de la media de los chicos, debido a la diferencia en los puntajes de la plancha, pero a pesar de ello no se aprecian diferencias significativas.

Tabla 6. Dominio B. Competencia física

\begin{tabular}{lcccc}
\hline & & Chicos & Chicas & p \\
\hline N (\%) & & $15(50)$ & $15(50)$ & \\
\hline Plancha & Media (DT) & $6,50(2,97)$ & $8,20(2,86)$ & \multirow{2}{*}{$0,119^{*}$} \\
\cline { 2 - 4 } (puntos) & Mediana (RI) & $6,50(5,00)$ & $10,00(4,00)$ & \\
\hline PACER & Media (DT) & $9,37(1,50)$ & $7,06(1,73)$ & \multirow{2}{*}{$0,345^{*}$} \\
\cline { 2 - 4 } (puntos) & Mediana (RI) & $10,50(2,50)$ & $7,00(3,00)$ & \\
\hline \multirow{2}{*}{ Total (puntos) } & Media (DT) & $24,80(5,00)$ & $26,20(5,78)$ & \\
\cline { 2 - 4 } & Mediana (RI) & $25,50(7,50)$ & $30,00(6,00)$ & \\
\hline
\end{tabular}

PACER (Progressive Aerobic Cardiovascular Endurance Run); Plancha (de 1 a 10 puntos); PACER (de 1 a 10 puntos); Total (1 a 30 puntos) Los datos se muestran como media (Desviación Típica) y Mediana (Rango Intercuartílico); Las diferencias de sexo fueron analizadas según el test de U Mann-Whitney U para las variables no paramétricas ( $\left.p^{\star}\right)$. 
La tabla 7, muestra las puntuaciones medias para el dominio motivación y confianza con las diferentes pruebas sobre motivación y confianza respondiendo a motivación intrínseca: ¿por qué eres activo/a?; competencia de actividad física: ¿cómo te sientes siendo activo/a? y predilección y adecuación: ¿qué se parece más a mí? Podemos comprobar que las medias son similares en ambos sexos, incluso la media total del dominio también es semejante en ambos sexos.

Tabla 7. Dominio C. Motivación y confianza

\begin{tabular}{|c|c|c|c|c|}
\hline & & Chicos & Chicas & $p$ \\
\hline $\mathrm{N}(\%)$ & & $19(48,72)$ & $20(51,28)$ & \\
\hline \multirow{2}{*}{ Parte 1} & Media (DT) & $7,11(0,77)$ & $7,13(0,61)$ & \multirow{2}{*}{$0,647^{*}$} \\
\hline & Mediana (RI) & $7,50(0)$ & $7,50(0,7)$ & \\
\hline \multirow{2}{*}{ Parte 2} & Media (DT) & $6,63(1,11)$ & $6,77(1,09)$ & \multirow{2}{*}{$0,813^{*}$} \\
\hline & Mediana (RI) & $6,88(1,4)$ & $6,90(0,7)$ & \\
\hline \multirow{2}{*}{ Parte 3} & Media (DT) & $6,66(0,97)$ & $6,92(0,73)$ & \multirow{2}{*}{$0,428^{*}$} \\
\hline & Mediana (RI) & $7,00(2,00)$ & $7,25(1,37)$ & \\
\hline \multirow{2}{*}{ Parte 4} & Media (DT) & $6,87(1,28)$ & $6,42(0,92)$ & \multirow{2}{*}{$0,184^{*}$} \\
\hline & Mediana (RI) & $7,50(1,5)$ & $6,50(1,87)$ & \\
\hline \multirow{2}{*}{ Total (puntos) } & Media (DT) & $27,28(2,95)$ & $27,25(2,33)$ & \multirow{2}{*}{$0,813^{*}$} \\
\hline & Mediana (RI) & $28,50(4,7)$ & $27,30(4,65)$ & \\
\hline \multicolumn{5}{|c|}{$\begin{array}{l}\text { Parte 1: motivación intrínseca (de } 1 \text { a } 7,5 \text { puntos); Parte 2: competición (de 1 a 7,5 puntos); Parte 3: predilección (de } 1 \text { a 7,5 } \\
\text { puntos); Parte 4: adecuación (1 a 7,5 puntos); Total dominio: de } 1 \text { a } 30 \text { puntos. Los datos se muestran como media } \\
\text { (Desviación Típica) y Mediana (Rango Intercuartílico); Las diferencias de sexo fueron analizadas según el test de U Mann- } \\
\text { Whitney U para las variables no paramétricas }\left(p^{*}\right)\end{array}$} \\
\hline
\end{tabular}

La tabla 8, muestra las puntuaciones medias para el dominio conocimiento y comprensión, teniendo en cuenta las diferentes preguntas sobre sus mejores habilidades. Podemos comprobar que en todos los puntajes la media de las chicas es superior a los chicos aunque la diferencia es mínima. Por lo que, en el total del dominio, son las chicas las cuales han obtenido mayor puntaje debido a la puntuación de cada una de estas 5 preguntas de conocimiento y comprensión. 
Tabla 8. Dominio D. Conocimiento y comprensión

\begin{tabular}{|c|c|c|c|c|}
\hline & & Chicos & Chicas & $P^{*}$ \\
\hline N (\%) & & $19(48,72)$ & $20(51,28)$ & \\
\hline \multirow{2}{*}{ Pregunta 1 (puntos) } & Media (DT) & $0,47(0,51)$ & $0,65(0,49)$ & \multirow{2}{*}{0,351} \\
\hline & Mediana (RI) & $0,00(1,00)$ & $1,00(1,00)$ & \\
\hline \multirow{2}{*}{ Pregunta 2 (puntos) } & Media (DT) & $0,89(0,31)$ & $0,90(0,31)$ & \multirow{2}{*}{1,00} \\
\hline & Mediana (RI) & $1,00(0,00)$ & $1,00(0,00)$ & \\
\hline \multirow{2}{*}{ Pregunta 3 (puntos) } & Media (DT) & $0,84(0,37)$ & $0,95(0,22)$ & \multirow{2}{*}{0,569} \\
\hline & Mediana (RI) & $1,00(0,00)$ & $1,00(0,00)$ & \\
\hline \multirow{2}{*}{ Pregunta 4 (puntos) } & Media (DT) & $0,26(0,45)$ & $0,40(0,50)$ & \multirow{2}{*}{0,478} \\
\hline & Mediana (RI) & $0,00(1,00)$ & $0,00(1,00)$ & \\
\hline \multirow{2}{*}{ Pregunta 5 (puntos) } & Media (DT) & $4,05(1,71)$ & $4,25(1,12)$ & \multirow{2}{*}{0,708} \\
\hline & Mediana (RI) & $4,00(3,00)$ & $4,00(2,00)$ & \\
\hline \multirow{2}{*}{ Total (puntos) } & Media (DT) & $6,53(2,09)$ & $7,15(1,31)$ & \multirow{2}{*}{0,365} \\
\hline & Mediana (RI) & $7,00(3,00)$ & $7,00(2,00)$ & \\
\hline
\end{tabular}

Pregunta 1: ¿Cuántos minutos de cada día deberías tú y los demás niños/as hacer actividades físicas que hagan que vuestro corazón lata más deprisa y os haga respirar más rápido, como caminar rápido y correr? Calcula el tiempo que deberías estar activo en el colegio y también cuando estás en casa o en tu barrio (de 0 a 1 punto); Pregunta 2: Hay muchos tipos de aptitudes físicas. Una se llama resistencia, o capacidad aeróbica, o acondicionamiento cardiorrespiratorio. El acondicionamiento cardiorrespiratorio significa: (de 0 a 1 punto); Pregunta 3: La fuerza muscular o resistencia muscular significa: (de 0 a 1 punto); Pregunta 4: Si quisieras mejorar en una habilidad deportiva (como darle una patada a un balón o atraparlo), ¿Cuál sería la mejor cosa que podrías hacer? (de 0 a 1 punto); Pregunta 5: Elige las palabras del recuadro para rellenar las palabras que faltan en la historia (de 1 a 6 puntos); Total (de 1 a 10 puntos); Los datos se muestran como media (Desviación Típica) y Mediana (Rango Intercuartílico); Las diferencias de sexo fueron analizadas según el test de $U$ Mann-Whitney $U$ para las variables no paramétricas $\left(p^{\star}\right)$.

La tabla 9, muestra los porcentajes de cada dominio y el total de los mismos con respecto a cada categoría de interpretación (insuficiente, en progreso, suficiente, excelente). Podemos comprobar cómo en los dominios de actividad diaria y conocimiento y comprensión, la mayoría de los participantes se encuentran en la categoría en progreso con un porcentaje mayor $52,9 \%$ y 36,8\% respectivamente; mientras que en los dominios correspondientes a competencia física y motivación y confianza, la mayoría se encuentra en la categoría excelente con un porcentaje de $70 \%$ y $78,9 \%$ respectivamente. 
Tabla 9. Puntación de dominios por categoría de interpretación en función del sexo y edad.

\begin{tabular}{lccccc}
\hline & $\mathrm{N}$ & $\begin{array}{c}\text { Insuficiente } \\
\mathrm{N}(\%)\end{array}$ & $\begin{array}{c}\text { En progreso } \\
\mathrm{N}(\%)\end{array}$ & $\begin{array}{c}\text { Suficiente } \\
\mathrm{N}(\%)\end{array}$ & $\begin{array}{c}\text { Excelente } \\
\mathrm{N}(\%)\end{array}$ \\
\hline Dominio A. Actividad diaria & 34 & $2(5,9)$ & $18(52,9)$ & $8(23,5)$ & $6(17,6)$ \\
\hline Dominio B. Competencia física & 30 & $2(6,7)$ & $2(6,7)$ & $5(16,7)$ & $21(70,00)$ \\
\hline $\begin{array}{l}\text { Dominio C. Motivación y } \\
\text { confianza }\end{array}$ & 38 & $0(0,0)$ & $4(10,5)$ & $4(10,5)$ & $30(78,9)$ \\
\hline $\begin{array}{l}\text { Dominio D. Conocimiento y } \\
\text { comprensión }\end{array}$ & 38 & $7(18,4)$ & $14(36,8)$ & $8(21,1)$ & $9(23,7)$ \\
\hline Total CAPL-2 & 30 & $0(0,0)$ & $6(20,0)$ & $2(6,7)$ & $22(73,3)$ \\
\hline
\end{tabular}

CAPL-2 (Canadian Assessment of Physicial Literacy) Los datos se muestran como N (\%)

El dominio de conocimiento y comprensión, así como el dominio de actividad diaria son los dos que se encuentran más repartidos entre las diferentes categorías.

Respecto al dominio de motivación y confianza podemos destacar que el porcentaje es 0\%, es decir ningún participante se encuentra en el nivel más bajo de este dominio.

En el total de CAPL.2 (Canadian Assessment of Physicial Literay) habiendo conocido los porcentajes finales de los dominios a través de las puntuaciones asignadas a cada uno de ellos podemos visualizar como el 73,3\% de los participantes se encuentra en la categoría excelente, mientras que el 20\% se encuentra en proceso y un 6,7\% suficiente, no encontrando ningún participante en el nivel insuficiente.

Siguiendo la tabla 9 que nos muestra los porcentajes de cada dominio por categoría de interpretación con respecto a los resultados de los estudios canadienses (Longmuir et al., 2015) se realizan diferentes comparaciones con respecto a nuestro estudio piloto.

Aunque las muestras utilizadas en dicho estudio exceden por muchos más participantes a las incluidas en nuestro estudio, podemos realizar comparaciones entre los canadienses y extremeños con respecto a su alfabetización física.

1. Dominio A o dominio actividad diaria

Si tenemos en cuenta el dominio A o dominio de actividad diaria se pudo comprobar cómo tanto en la evaluación canadiense como en la evaluación de la alfabetización física en niños/as extremeños, la mayoría de ellos se encuentra en la categoría de progreso en torno al 53\%. El 
menor número de alumnos se encuentra en la categoría insuficiente siendo el porcentaje de los canadienses un 3\% mientras que el porcentaje español refiere un 5,9\%.

2. Dominio B o dominio de competencia física

Con respecto al dominio B o dominio de competencia física los niños/as extremeños poseen un mayor nivel en este dominio encontrándose la mayoría en un porcentaje en torno al 70\% mientras los alumnos canadienses están más repartidos entre las categorías en progreso y suficiente.

\section{Dominio C o dominio de motivación y confianza}

Ahora bien, como se ha comentado anteriormente, el dominio motivación y confianza tiene relación con el dominio de competencia física y por ello la mayoría de los extremeños también se encuentra en el nivel excelente con un 78,9\% mientras que los alumnos canadienses en este dominio también están repartidos entre las categorías en progreso y suficiente. Debido a esta relación tanto en un estudio como en otro, podemos comprobar cómo ambas partes de la alfabetización física (competencia física y motivación) estarían totalmente relacionadas.

\section{Dominio D o dominio de conocimiento y comprensión}

El domino D o dominio de conocimiento y comprensión, nos muestra cómo los alumnos están totalmente repartidos entre las tres categorías (insuficiente, en progreso, suficiente, excelente) contando con un mayor número de alumnos en la categoría en progreso al igual que los canadienses con un 59\% de los niños/as participantes en el estudio.

Para finalizar esta comparación, pudimos observar cómo la mayoría de los alumnos extremeños se encuentran en la categoría excelente con un porcentaje de 73,3\%, el 20\% de alumnos en la categoría de progreso y un 6,7\% en la categoría suficiente, mientras que la mayoría de los alumnos canadienses se encuentran en la categoría en progreso con un porcentaje del 47\%, un 34\% en la categoría suficiente, 3\% insuficiente y un 16\% en la categoría más alta (excelente) Por lo que, con lo comprobado en nuestro estudio podríamos decir que los alumnos extremeños poseen mayor alfabetización física que los canadienses, aunque siempre tener en cuenta que se usaron muestras de participantes mucho más pequeñas en extremeños que canadienses. 


\section{Discusión}

Con base a los resultados obtenidos, se pudo afirmar que no existían diferencias estadísticamente significativas entre sexos en ninguna de las pruebas llevadas a cabo para calcular la alfabetización física de niños entre 8 y 12 años en Extremadura.

Los resultados obtenidos en este estudio, revelaron que los datos generados de esta del CAPL-2 para los niños de 8 a 12 años son en gran medida consistentes con el modelo hipotético CAPL-2 (Francis et al., 2016) y la definición internacional de alfabetización física (Whitehead, 2016). De acuerdo con la definición nacional, el modelo apoyó la participación en actividad física (actividad física y comportamiento sedentario en vida cotidiana) competencia física (aptitud física de los participantes y habilidad de movimiento), motivación, comprensión y conocimiento para diferentes factores.

Una variable importante que determina el rendimiento es la motivación del adolescente hacia el proceso de aprendizaje, siendo este un dominio dentro de este estudio. El concepto de motivación educativa ha ido evolucionando desde una perspectiva más cuantitativa (teorías basadas en la reducción o ampliación del impulso) hacia otras más cualitativas, centradas en la interacción entre el estilo del alumno para aprender y los condicionantes de la propuesta educativa (Cavero, 2006). Se pudo apreciar cómo cuando el porcentaje de alumnos con mayor motivación y confianza fue elevado (78,9\%), el dominio de competencia física también resultó con porcentajes altos (73,3\%), tanto es nuestro estudio, como en niños canadienses (Longmuir et al., 2015). En esta línea, de los estudios sobre motivación para participar en la actividad física durante la etapa de iniciación se desprende que los motivos valorados como más importantes son "mejorar las propias destrezas o habilidades físicas", "divertirse", "la emoción y el reto propio de la actividad", "estar en forma" y, también "estar con los amigos y hacer nuevas amistades" (un motivo social de carácter afiliativo) (Weinberg et al., 2000). Numerosos estudios abordan la influencia de la motivación sobre el nivel de actividad física (Moreno Murcia, Borges Silva, Marcos Pardo, Sierra Rodríguez, \& Huéscar Hernández, 2012; Solomon-Moore et al., 2017; Teixeira, Carraça, Markland, Silva, \& Ryan, 2012) de manera que muchas intervenciones de los padres se pueden identificar como valor personal hacia el ejercicio, de manera que motivan el comportamiento de sus hijos/as hacia su práctica desde las edades más tempranas (De Meyer et al., 2016). Además, algunos estudios mostraron que los estudiantes físicamente activos obtienen una puntuación más alta en motivación autónoma y menos motivación que los estudiantes no activos (Sánchez-Oliva et al., 2020; 
Viira \& Koka, 2012). Estudiantes que satisfacían las necesidades de autonomía, competencia, y la relación, son más propensos a participar en actividades relacionados con la clase de educación física por motivos autodeterminados y que también es menos probable que se sientan desmotivados (Sánchez-Oliva et al., 2020).

Con respecto a la competencia física, se pudo comprobar cómo no existían diferencias significativas entre ambos sexos, esto puede deberse a ya que la mayoría de las capacidades físicas básicas tiene un desarrollo acentuado al inicio de la pubertad, más específicamente a partir de los 11 años (Eras Ordoñez, 2015; Muñoz Rivera, 2009). Más específicamente sobre la resistencia se han realizado investigaciones en el contexto escolar, en las cuales se señaló que las chicas obtenían mejores valores que los chicos hasta los 12 años, al igual que en velocidad, y es a partir de esta cuando empiezan a invertirse los valores debido a un estancamiento por parte del sexo femenino (Gallego, Sánchez, Vacas, \& Zagalaz, 2016; Vallejo Cuéllar, 2004). Por tanto la falta diferencias significativas puede deberse a que no es hasta los 12 años en niñas o 14 en niños (Vargas, 1991), cuando empieza una gran fase de diferenciación por sexo, donde se podrían producir grandes mejoras en capacidades y habilidades motoras debidas a la maduración anatomofisiológica, es decir, crecimiento en altura, extremidades o incremento de masa muscular, lo cual debería llevar a una estabilidad en el desarrollo de las habilidades motoras, y su mejora, debida también a experiencias y aprendizajes previos. Sim embargo, diversos estudios si encuentran diferencias significativas para este rango de edad en estudios con un tamaño muestral mayor (Secchi, García, España-Romero, \& Castro-Piñero, 2014). En este sentido, el CAPL-2 en adolescente de entre 12 y 16 años (Blanchard, Van Wyk, Ertel, Alpous, \& Longmuir, 2019), si se obtuvieron diferencias significativas entre sexos, así como otros estudios que han evaluado condición física en muestra adolescente (Castro-Piñero et al., 2009; Mendoza-Muñoz et al., 2020; Ortega et al., 2011; Ortega et al., 2008; Ortega et al., 2005; Rosa Guillamón, Rodríguez García, García Cantó, \& Pérez Soto, 2015). En general, la mayoría de estudios ponen en concordancia como los niveles de fuerza, velocidad/agilidad, rendimiento y capacidad aeróbica son mayores en los participantes masculinos (Secchi et al., 2014), así como que los niños con alta condición física cardiovascular muestran menores valores de índice de masa corporal (IMC), porcentaje de grasa corporal, o más específicamente en la batería de tests ALPHA-Fitness mejores niveles en saltos de longitud, en la prueba Course Navette o velocidad 4x 10 metros (Mayorga-Vega, Merino-Marban, \& Rodríguez-Fernández, 2013; Mendoza-Muñoz et al., 2020), donde además presenta una clara relación con la salud cardiovascular de los niños (Mayorga-Vega et al., 2013; Ruíz et al., 2011). 
Por tanto, debido a que dicha batería tiene pruebas en común o similares a las desarrolladas en esta evaluación podría establecerse como línea de futuro la relación de esta evaluación con la salud cardiovascular, con el fin de apoyar, ampliar y/o contrastar estos resultados.

En relación a las pautas de actividad física y comportamiento diario para niños de 5 a 17 años de edad, la OMS (OMS, 2010) recomienda que se acumulen por lo menos 60 minutos de actividad física de intensidad moderada o vigorosa o Moderate to vigorous physical activity (MVPA). Colley, Janssen \& Tremblay sugieren que 12.000 pasos al día equivalen al menos 60 minutos de MVPA. President's Council on Physical Fitness and Sports recomienda 12.000 pasos para las niñas y 15.000 pasos para los niños (Colley, Janssen, \& Tremblay, 2012). En este sentido se puede decir que la mayoría de participantes del estudio no alcanzan las recomendaciones de pasos, ya que la puntuación medida obtenida es de 17,31 $( \pm 4,55)$ sobre 25 en el caso de los chicos y 14,30 $( \pm 6,07)$ en el caso de las chicas, lo que equivale a una media diaria de pasos de 12738 y 11131 respectivamente, localizándose así más del 50\% de la muestra en los niveles más bajos de actividad diaria (Tabla 9).

Más específicamente, para poder comparar los datos de este estudios con sus análogo canadienses, en la tabla 10 (Longmuir et al., 2015) se puede observar la puntación de estos, en los diferentes dominios por categoría de interpretación. Aunque las muestras utilizadas este estudio (Longmuir et al., 2015) exceden por muchos más participantes a las incluidas en el presente estudio, podemos realizar comparaciones entre los ellos y la muestra extremeña con respecto a su alfabetización física para obtener un punto de referencia en torno a los valores normativos canadienses respecto a la muestra estudiada.

Si tenemos en cuenta el dominio A o dominio de actividad diaria se pudo comprobar cómo tanto en la evaluación canadiense como en la evaluación de la alfabetización física en extremeños, la mayoría de ellos se encuentra en la categoría de progreso en torno al 53\%. El menor número de alumnos se encuentra en la categoría insuficiente siendo el porcentaje de los canadienses un 5\% mientras que el porcentaje español refiere un 5,9\%.

Con respecto al dominio B o dominio de competencia física los niños/as extremeños poseen un mayor nivel que los canadienses, encontrándose la un 70\% en el nivel excelente, mientras los canadienses están más repartidos entre las categorías en progreso y suficiente (61 y 23\% respectivamente).

En relación con dominio de motivación y confianza podemos observar cómo un 78,9\% de los niños extremeños alcanzan un nivel excelente, mientras que los alumnos canadienses en este dominio también están repartidos entre las categorías en progreso y suficiente (59 y 31\% respectivamente). Debido a esta relación tanto en un estudio como en otro, podemos 
comprobar cómo ambas partes de la alfabetización física (competencia física y motivación) podría estar relacionadas.

Tabla 10. Puntación de dominios por categoría de interpretación.

\begin{tabular}{|c|c|c|c|c|c|}
\hline & $\mathrm{N}$ & $\begin{array}{l}\text { Insuficiente } \\
\qquad N(\%)\end{array}$ & $\begin{array}{c}\text { En } \\
\text { progreso } \\
\mathrm{N}(\%)\end{array}$ & $\begin{array}{l}\text { Suficiente } \\
\text { N (\%) }\end{array}$ & $\begin{array}{l}\text { Excelente } \\
N(\%)\end{array}$ \\
\hline $\begin{array}{l}\text { Dominio A. Actividad } \\
\text { diaria }\end{array}$ & 702 & $38(5)$ & 335 (48) & 214(31) & $115(16)$ \\
\hline $\begin{array}{l}\text { Dominio B. } \\
\text { Competencia física }\end{array}$ & 691 & $44(6)$ & $418(61)$ & $160(23)$ & $69(10)$ \\
\hline $\begin{array}{l}\text { Dominio C. Motivación } \\
\text { y confianza }\end{array}$ & 740 & $56(8)$ & 434(59) & 232(31) & $18(2)$ \\
\hline $\begin{array}{l}\text { Dominio D. } \\
\text { Conocimiento y } \\
\text { comprensión }\end{array}$ & 687 & $24(3)$ & $323(47)$ & 231(34) & 109(16) \\
\hline Total CAPL-2 & 403 & $12(3)$ & $212(53)$ & $117(29)$ & $62(15)$ \\
\hline \multicolumn{6}{|c|}{$\begin{array}{l}\text { CAPL-2 (Canadian Assessment of Physicial Literacy) Los datos se muestran como N (frecuencia) y \% } \\
\text { (porcentaje válido). } \\
\text { Nota. Recuperado de "The Canadian Assessment of Physical Literacy: methods for children in grades } 4 \text { to } \\
6 \text { (8 to } 12 \text { years)", Longmuir, P. E., Boyer, C., Lloyd, M., Yang, Y., Boiarskaia, E., Zhu, W., \& Tremblay, M. S., } \\
\text { 2015, BMC Public Health, 15(1). }\end{array}$} \\
\hline
\end{tabular}

El domino D o dominio de conocimiento y comprensión, nos muestra cómo los alumnos están totalmente repartidos entre las tres categorías (insuficiente, en progreso, suficiente, excelente) contando con un mayor número de alumnos en la categoría en progreso al igual que los canadienses con un $47 \%$.

Para finalizar esta comparación, se puede observar como la mayoría de los alumnos extremeños se encuentran en la categoría excelente con un porcentaje de $73,3 \%$, el $20 \%$ de alumnos en la categoría de progreso y un 6,7\% en la categoría suficiente, mientras que la mayoría de los alumnos canadienses se encuentran en la categoría en progreso con un porcentaje del 53\%, un 29\% en la categoría suficiente, 3\% insuficiente y un 15\% en la categoría más alta (excelente). Por lo que, los resultados nos muestran que la muestra de alumnos extremeños estudiados posee mayor alfabetización física que los canadienses, aunque 
siempre debemos tener en cuenta que se usaron muestras de participantes mucho más pequeñas que en los canadienses.

Este estudio piloto presenta ciertas limitaciones, las cuales deben ser tenidas en cuenta a la hora de interpretar y analizar los resultados y conclusiones de este. La principal limitación es la falta de muestra de este, ya que el tamaño se vio limitado debido a la situación generada por la pandemia mundial (COVID-19). En relación con la anterior y por falta de tiempo debido al cierre de los centros educativos, la ausencia de la realización de una de las pruebas perteneciente al dominio de competencia física, la prueba CAMSA. No obstante, como se recoge en el desarrollo del presente documento, esta interpretación se ha realizado teniendo en cuenta el apartado de "datos perdidos" referenciado en el manual original (HALO).

Finalmente, también debemos destacar que la alfabetización física aún no ha sido muy estudiada y valorada en niños por lo que existen pocos estudios sobre este tema, lo que supone una limitación a la hora de recabar información y establecer comparaciones. Al mismo tiempo, esto supone una de las principales aportaciones de este estudio, ya que es el primero en evaluar la alfabetización física en población española.

Por tanto, una de las implicaciones de este estudio es resaltar la importancia y abrir una nueva línea de investigación debido a la necesidad de potenciar la alfabetización física en las edades más tempranas en las que tiene lugar el desarrollo motor. Su logro permitirá la participación en una actividad física estructurada plena, adoptando un estilo de vida activo a edades tempranas el cual puede continuar hasta la edad adulta.

\section{Conclusión}

Con base a los resultados obtenidos en el presente trabajo, se puede concluir que no existieron diferencias significativas entre sexos teniendo en cuenta los diferentes dominios, así como las pruebas dentro de cada uno de ellos. Más específicamente, en el dominio de actividades física diaria, se observa que los chicos presentan mayor puntuación que las chicas, aunque no se aprecian diferencias significativas. Sin embargo, en el dominio de competencia física la puntuación es mayor para las chicas, pero sin apreciarse igualmente diferencias significativas. Respecto al dominio de motivación y confianza se puede observar puntuaciones muy similares para ambos sexos. Y finalmente, para el ultimo dominio conocimiento y compresión, se puede comprobar como la puntuación de las chicas se encuentra por encima de los chicos, pero como los anteriores dominios, sin establecerse diferencias significativas. 
Además, se pudo afirmar que los niveles de alfabetización física en la población extremeña son mayores que sus análogos canadienses.

La evaluación canadiense de alfabetización física ofrece una evaluación integral del compromiso de actividad física, competencia física, motivación y confianza y conocimiento y comprensión relacionados con el estilo de vida activos en niños de 8 a 12 años. El monitoreo de estas medidas puede ser muy relevantes para detectar y prevenir posibles problemas relacionados con el sedentarismo y por consiguiente los asociados al mismo.

\section{Agradecimientos}

Los autores desean agradecer a las escuelas y profesionales que permitieron el acceso para llevar a cabo este estudio.

\section{Conflicto de interés}

Los autores declaran no tener conflicto de interés.

\section{Financiación}

Esta investigación está financiada por el Servicio Público de Empleo de Extremadura (SEXPE), número de subvención TE-0009-18. Los financiadores no desempeñaron ningún papel en el diseño del estudio, la decisión de publicar o la preparación del manuscrito.

\section{Referencias}

Blanchard, J., Van Wyk, N., Ertel, E., Alpous, A., \& Longmuir, P. (2019). Canadian Assessment of Physical Literacy in grades 7-9 (12-16 years): Preliminary validity and descriptive $\begin{array}{llll}\text { results. Journal of Sport } & \text { Sciences, }\end{array}$ https://doi.org/10.1080/02640414.2019.1689076

Bonilla, A. M. P. (2016). Impacto de la clase de educación física sobre la actividad moderada y vigorosa en niños de primaria. Revista Mexicana de Investigación en Cultura Física y Deporte, 1(1), 150-173.

Boyer, C., Tremblay, M., Saunders, T., McFarlane, A., Borghese, M., Lloyd, M., \& Longmuir, P. (2013). Feasibility, validity, and reliability of the plank isometric hold as a field-based assessment of torso muscular endurance for children 8-12 years of age. Pediatr Exerc Sci, 25(3), 407-422. https://doi.org/10.1123/pes.25.3.407 
Broc Cavero, M. Á. (2006). Motivación y rendimiento académico en alumnos de Educación Secundaria Obligatoria y Bachillerato LOGSE. Revista de educación.

Cairney, J., Dudley, D., Kwan, M., Bulten, R., \& Kriellaars, D. (2019). Physical literacy, physical activity and health: Toward an evidence-informed conceptual model. Sports Medicine, 49(3), 371-383. https://doi.org/10.1007/s40279-019-01063-3

Cairney, J., \& Veldhuizen, S. (2017). Organized sport and physical activity participation and body mass index in children and youth: A longitudinal study. Preventive Medicine Reports, 6, 336-338. https://doi.org/10.1016/j.pmedr.2017.04.005

Capdevila Ortís, L., Niñerola i Maymì, J., \& Pintanel i Bassets, M. (2004). Motivación y actividad física: el autoinforme de motivos para la práctica de ejercicio físico (AMPEF). Revista de psicología del Deporte, 13(1), 0055-0074.

Castro-Piñero, J., González-Montesinos, J. L., Mora, J., Keating, X. D., Girela-Rejón, M. J., Sjöström, M., \& Ruiz, J. R. (2009). Percentile values for muscular strength field tests in children aged 6 to 17 years: influence of weight status. The Journal of Strength \& Conditioning Research, 23(8), 2295-2310. https://doi.org/10.1519/JSC.0b013e3181b8d5c1

Colley, R. C., Carson, V., Garriguet, D., Janssen, I., Roberts, K. C., \& Tremblay, M. S. (2017). Physical activity of Canadian children and youth, 2007 to 2015: Statistics Canada.

Colley, R. C., Janssen, I., \& Tremblay, M. S. (2012). Daily step target to measure adherence to physical activity guidelines in children. Medicine \& Science in Sports \& Exercise, 44(5), 977-982. https://doi.org/10.1249/MSS.0b013e31823f23b1

de Balazs, A. C. R., de D'Amico, R. L., \& Cedeño, J. J. M. (2017). Alfabetización física: una percepción reflexiva. Dialógica: revista multidisciplinaria, 14(1), 87-102.

De Meyer, J., Soenens, B., Vansteenkiste, M., Aelterman, N., Van Petegem, S., \& Haerens, L. (2016). Do students with different motives for physical education respond differently to autonomy-supportive and controlling teaching? Psychology of Sport and Exercise, 22, 72-82. https://doi.org/10.1016/j.psychsport.2015.06.001

Eras Ordoñez, L. F. (2015). Desarrollo de las capacidades físicas según las etapas de crecimiento y evolución fisiológica del ser humano.

Francis, C. E., Longmuir, P. E., Boyer, C., Andersen, L. B., Barnes, J. D., Boiarskaia, E., .. Hands, B. P. (2016). The Canadian assessment of physical literacy: development of a model of 
children's capacity for a healthy, active lifestyle through a Delphi process. Journal of Physical Activity and Health, 13(2), 214-222. https://doi.org/10.1123/jpah.2014-0597

Gallego, F. L., Sánchez, A. J. L., Vacas, N. E., \& Zagalaz, J. C. (2016). Influencia del género, la edad y el nivel de actividad física en la condición física de alumnos de educación primaria. Revisión Bibliográfica. Retos: nuevas tendencias en educación física, deporte y recreación (29), 129-133.

García, C. M., \& González-Jurado, J. A. (2017). Impacto de la inactividad física en la mortalidad y los costos económicos por defunciones cardiovasculares: evidencia desde Argentina. Revista Panamericana de Salud Pública, 41, e92. https://doi.org/10.26633/RPSP.2017.92

Gómez, S. F., Lorenzo, L., Ribes, C., \& Homs, C. (2019). Informe estudio PASOS 2019. Retrieved from Sant Boi de Llobregat, Barcelona

HALO. Obesity Research Group (HALO), Canadian Assessment of Physical Literacy: Manual for Test Administration. 2014.

Iraheta, B. E., \& Bogantes, C. Á. (2020). Análisis del sobrepeso y obesidad, niveles de actividad física y autoestima de la niñez salvadoreña. MHSalud, 17(1), 1-18. https://doi.org/10.15359/mhs.17-1.1

Longmuir, P. E., Boyer, C., Lloyd, M., Borghese, M. M., Knight, E., Saunders, T. J., ... Tremblay, M. S. (2017). Canadian Agility and Movement Skill Assessment (CAMSA): Validity, objectivity, and reliability evidence for children 8-12 years of age. J Sport Health Sci, 6(2), 231-240. https://doi.org/10.1016/j.jshs.2015.11.004

Longmuir, P. E., Boyer, C., Lloyd, M., Yang, Y., Boiarskaia, E., Zhu, W., \& Tremblay, M. S. (2015). The Canadian assessment of physical literacy: methods for children in grades 4 to 6 (8 to 12 years). BMC public health, 15(1), 767. https://doi.org/10.1186/s12889-015-2106-6

Longmuir, P. E., Gunnell, K. E., Barnes, J. D., Belanger, K., Leduc, G., Woodruff, S. J., \& Tremblay, M. S. (2018). Canadian Assessment of Physical Literacy Second Edition: a streamlined assessment of the capacity for physical activity among children 8 to 12 years of age. BMC public health, 18(2), 1047. https://doi.org/10.1186/s12889-018-5902-y

Longmuir, P. E., \& Tremblay, M. S. (2016). Top 10 research questions related to physical literacy. Research Quarterly for Exercise and Sport, 87(1), 28-35. https://doi.org/10.1080/02701367.2016.1124671 
Longmuir, P. E., Woodruff, S. J., Boyer, C., Lloyd, M., \& Tremblay, M. S. (2018). Physical Literacy Knowledge Questionnaire: feasibility, validity, and reliability for Canadian children aged 8 to 12 years. BMC public health, 18(2), 1035. https://doi.org/10.1186/s12889-0185890-y

Martínez-Vizcaíno, V., \& Sánchez-López, M. (2008). Relación entre actividad física y condición física en niños y adolescentes. Revista española de cardiología, 61(2), 108-111. https://doi.org/10.1157/13116196

Mayorga-Vega, D., Merino-Marban, R., \& Rodríguez-Fernández, E. (2013). Relación entre la capacidad cardiorrespiratoria y el rendimiento en los tests de condición física relacionada con la salud incluidos en la batería ALPHA en niños de 10-12 años. Cultura, Ciencia y Deporte, 8(22), 41-47. https://doi.org/10.12800/ccd.v8i22.222

Mendoza-Muñoz, M., Adsuar, J. C., Pérez-Gómez, J., Muñoz-Bermejo, L., Garcia-Gordillo, M. Á., \& Carlos-Vivas, J. (2020). Influence of body composition on physical fitness in adolescents. Medicina, 56(7), 328. https://doi.org/10.3390/medicina56070328

Ministerio de Sanidad Servicios Sociales e Igualdad, I. N. d. E. Encuesta Nacional de Salud España ENSE 2017. Ministerio de Sanidad Servicios Sociales e Igualdad: Madrid, Spain, 2018.

Moreno Murcia, J., Borges Silva, F., Marcos Pardo, P., Sierra Rodríguez, A., \& Huéscar Hernández, E. (2012). Motivación, frecuencia y tipo de actividad en practicantes de ejercicio físico.

Muñoz Rivera, D. (2009). Capacidades físicas básicas. Evolución, factores y desarrollo. Sesiones prácticas. Buenos Aires. Revista Digital, 14.

Organization, W. H. (2010). Recomendaciones mundiales sobre actividad física para la salud.

Ortega, F. B., Artero, E. G., Ruiz, J. R., España-Romero, V., Jiménez-Pavón, D., VicenteRodríguez, G., . . O. Ottevaere, C. (2011). Physical fitness levels among European adolescents: the HELENA study. British journal of sports medicine, 45(1), 20-29. https://doi.org/10.1136/bjsm.2009.062679

Ortega, F. B., Artero, E. G., Ruiz, J. R., Vicente-Rodriguez, G., Bergman, P., Hagströmer, M., . . . Rey-Lopez, J. (2008). Reliability of health-related physical fitness tests in European adolescents. The HELENA Study. International Journal of Obesity, 32(5), S49-S57. https://doi.org/10.1038/ijo.2008.183 
Ortega, F. B., Ruiz, J. R., Castillo, M. J., Moreno, L. A., González-Gross, M., Wärnberg, J., . . . Group, A. (2005). Low level of physical fitness in Spanish adolescents. Relevance for future cardiovascular health (AVENA study). Revista Española de Cardiología (English Edition), 58(8), 898-909. https://doi.org/10.1016/S1885-5857(06)60372-1

Pérez-Farinós, N., López-Sobaler, A. M., Dal Re, M., Villar, C., Labrado, E., Robledo, T., \& Ortega, R. M. (2013). The ALADINO study: a national study of prevalence of overweight and obesity in Spanish children in 2011. BioMed Res Int, 2013. https://doi.org/10.1155/2013/163687

Roetert, E. P., \& Jefferies, S. C. (2014). Embracing physical literacy. Journal of Physical Education, Recreation and Dance, 85(8), 38-40. https://doi.org/10.1080/07303084.2014.948353

Roetert, E. P., Kriellaars, D., Ellenbecker, T. S., \& Richardson, C. (2017). Preparing students for a physically literate life. Journal of Physical Education, Recreation \& Dance, 88(1), 5762. https://doi.org/10.1080/07303084.2017.1252554

Roetert, E. P., \& MacDonald, L. C. (2015). Unpacking the physical literacy concept for K-12 physical education: What should we expect the learner to master? Journal of Sport and Health Science, 4(2), 108-112. https://doi.org/10.1016/j.jshs.2015.03.002

Rosa Guillamón, A., Rodríguez García, P. L., García Cantó, E., \& Pérez Soto, J. J. (2015). Niveles de condición física de escolares de 8 a 11 años en relación al género ya su estatus corporal.

Ruíz, J. R., España Romero, V., Castro Piñero, J., Artero, E. G., Ortega, F., Cuenca García, M., . . Mora, J. (2011). Batería ALPHA-Fitness: test de campo para la evaluación de la condición física relacionada con la salud en niños y adolescentes. Nutrición Hospitalaria, 26(6), 1210-1214.

Sánchez-Oliva, D., Mouratidis, A., Leo, F. M., Chamorro, J. L., Pulido-González, J. J., \& GarcíaCalvo, T. (2020). Understanding Physical Activity Intentions in Physical Education Context: A Multi-Level Analysis from the Self-Determination Theory. International Journal of Environmental Research and Public Health, 17(3), 799. https://doi.org/10.3390/ijerph17030799

Secchi, J. D., García, G. C., España-Romero, V., \& Castro-Piñero, J. (2014). Condición física y riesgo cardiovascular futuro en niños y adolescentes argentinos: una introducción de la batería ALPHA. Archivos argentinos de pediatría, 112(2), 132-140. https://doi.org/10.5546/aap.2014.132 
Solomon-Moore, E., Sebire, S. J., Thompson, J. L., Zahra, J., Lawlor, D. A., \& Jago, R. (2017). Are parents' motivations to exercise and intention to engage in regular family-based activity associated with both adult and child physical activity? BMJ open sport \& exercise medicine, 2(1). https://doi.org/10.1136/bmjsem-2016-000137

Spengler, J. O., \& Cohen, J. (2015). Physical literacy: A global environmental scan. Washington, DC: The Aspen Institute.

Teixeira, P. J., Carraça, E. V., Markland, D., Silva, M. N., \& Ryan, R. M. (2012). Exercise, physical activity, and self-determination theory: a systematic review. International journal of behavioral nutrition and physical activity, 9(1), 78. https://doi.org/10.1186/1479-5868$\underline{9-78}$

Tremblay, M. S., Colley, R. C., Saunders, T. J., Healy, G. N., \& Owen, N. (2010). Physiological and health implications of a sedentary lifestyle. Applied physiology, nutrition, and metabolism, 35(6), 725-740. https://doi.org/10.1139/H10-079

Tremblay, M. S., Longmuir, P. E., Barnes, J. D., Belanger, K., Anderson, K. D., Bruner, B., . . Hall, N. (2018). Physical literacy levels of Canadian children aged 8-12 years: descriptive and normative results from the RBC Learn to Play-CAPL project. BMC public health, 18(2), 1036. https://doi.org/10.1186/s12889-018-5891-x

Twisk, J., Kemper, H., \& Van Mechelen, W. (2000). Tracking of activity and fitness and the relationship with cardiovascular disease risk factors. Medicine \& Science in Sports \& Exercise, 32(8), 1455-1461. https://doi.org/10.1097/00005768-200008000-00014

Vallejo Cuéllar, L. (2004). Desarrollo de la condición física y sus efectos sobre el rendimiento físico y la composición corporal de niños futbolistas: Universitat Autònoma de Barcelona.

Vargas, N. (1991). Crecimiento y desarrollo. Texto de Pediatría.

Viira, R., \& Koka, A. (2012). Participation in afterschool sport: Relationship to perceived need support, need satisfaction, and motivation in physical education. Kinesiology: International journal of fundamental and applied kinesiology, 44(2), 199-208.

Vuik, S., Lerouge, A., Guillemette, Y., Feigl, A., \& Aldea, A. (2019). The economic burden of obesity. https://doi.org/10.1787/6cc2aacc-en

Weinberg, R., Tenenbaum, G., McKenzie, A., Jackson, S., Anshel, M., Grove, R., \& Fogarty, G. (2000). Motivation for youth participation in sport and physical activity: relationships 
to culture, self-reported activity levels, and gender. International Journal of Sport Psychology, 31, 321-346.

Whitehead, M. (2010). Physical literacy: Throughout the lifecourse: Routledge. https://doi.org/10.4324/9780203881903

Whitehead, M. (2016). International physical literacy association.

Wijnhoven, T. M., van Raaij, J. M., Spinelli, A., Starc, G., Hassapidou, M., Spiroski, I., . . . Hovengen, R. (2014). WHO European Childhood Obesity Surveillance Initiative: body mass index and level of overweight among 6-9-year-old children from school year 2007/2008 to school year 2009/2010. BMC public health, 14(1), 806. https://doi.org/10.1186/1471-2458-14-806 